\title{
Formulated Mathematical Model for Delayed Particle Flow in Cascaded Sub-Surface Water Reservoirs with Validation on River Flow
}

\author{
Ombaki Richard, ${ }^{1}+254727908185$, Kerongo Joash ${ }^{2},+25472547208$, Okwoyo M. James ${ }^{3},+254764602901$ \\ 1. Department of mathematics and actuarial science- (Kisii University); \\ 2. Department of mathematics and actuarial science- (Kisii University); \\ 3. School of mathematics -(University of Nairobi);
}

\begin{abstract}
Pollution of sub-surface water reservoirs mainly rivers and streams through contaminated water point sources (CWPS) was studied. The objective was to formulate a discrete time delay mathematical model which describes the dynamics of reservoir pollution using mixing-problem processes that involve single species contaminants such as nitrates, phosphorous and detergents. The concentration $x(t)$ of pollutants was expressed as a function of the inflow and outflow rates using the principle for the conservation of mass. Systems of ODEs generated from principles of mixing problems were refined into a system of DDEs so that the concentration of pollutant leaving the reservoir at time would be determined at some earlier instant, for the delay. The formulated model is a mathematical discrete time delay model which would be used to describe the dynamics of sub-surface water reservoir pollution. The results from the validation of the model were analyzed to determine how time delays in the mixing processes affect the rate of particle movement in water reservoirs.
\end{abstract}

Key words: Particle Flow, Sub-Surface Water, Reservoirs Pollution.

\section{Introduction}

In addition to groundwater, sub-surface water systems that include rivers, ponds, dams, and lakes are important water resource for municipalities, domestic, agriculture, and industry. Therefore, the capability to predict the levels of concentration and rate of movement of contaminants in the water resource is of vital importance for the reliable assessment of hazardous or risk arising from water contamination problems, and for the decision of efficient and effective techniques to mitigate them. Reliable and quantitative prediction of pollutants movement in water systems can be achieved only if we understand the processes controlling the migration of contaminants. These processes include advection, hydrodynamic dispersion, and physical, chemical and biological reactions that affect their soluble concentration in water systems (Nick, Foppen, Kulabako, Xavier, Samuel \& Wagner, 2012).

Reduction of sub-surface water pollution largely involves the scientific understanding of these processes which control the fate and movement of pollutants in the sub-surface environment. First, the advection transport involve movement of dissolved chemicals with the flowing water; second, hydrodynamic dispersion, involve molecular and ionic diffusion together with small-scale variations in the flow velocity through the porous media causing the paths of dissolved molecules and ions to diverge or spread from the average direction of water flow; third, reactions in which some amount of a particular dissolved chemical species (such as herbicides) may be removed from the water as a result of biological, chemical, and physical reactions in water or between the water and the solid aquifer materials or other liquid phases. Lastly, fluid point sources, where water of one composition is introduced into and mixed with water of a different composition. In addition, it is important to understand the most challenging problems associated with pollution of water systems, which include prevention of the introduction of contaminants in water systems; prediction of their movement if they are introduced; and methods of their removal, to some extent, in order to protect the biosphere effectively (Patil \& Chore, 2014).

The mathematical representation in the transport models to predict the contaminant migration in sub-surface water systems is one major area to supplement to the scientific knowledge on pollution processes. Formulation of the transport models involves determination of different parameters in the field and the laboratory using different methods and consequent applications of the established conceptual models for development of management measures to control and/or prevent the introduction of contaminants in water systems (EPA, 2007).

\section{Background}

Migration of dissolved (or undissolved) organic and inorganic substances into sub-surface water reservoirs through point sources, largely involve mixing processes within the system of flow. The mixing processes significantly alter the predictive rate of flow of pollutants in the water system, and therefore the time inherent in these processes need to be accounted for. The study considered changes in contaminant concentration which occur within dynamic sub-surface water systems of cascading water reservoirs due to fluid point sources, where water of one composition of pollutant is introduced into and mixed with water of a different composition. A numerical model for the process was formulated 
using mixing-problem analyzes in water tanks (Antonin, 2013). The purpose was to apply the model to predict pollution concentration attenuation in water systems and this may be helpful to approximate time inherent in the process of pollutants accumulation in water reservoirs.

\subsection{Continuity Equation}

\section{Governing Equations and Principles}

In water dynamical systems, development of mathematical equations to describe the groundwater flow and transport processes is achieved through the fundamental principle of mass conservation of fluid or solute. The general equation for conservation of mass may be represented in global variables, using a representative volume of porous medium as:

$$
Q_{\text {in }}-Q_{\text {out }}-Q_{\text {production }}=Q_{\text {Accumulation }}
$$

Where is the mass of fluid or solute. This is the equation for conservation of mass (or continuity equation) which may be combined with a mathematical expression of the relevant dynamic process to obtain a differential equation describing flow or transport (Anderson, Woessner,\& Hunt, 2016).

\subsection{Equations for Mixing-problem Processes in Cascade Reservoirs}

In modeling population dynamics, Antonin (2013) derives the following system of ODE from cascade arrangement of water tanks

$$
\begin{aligned}
& \frac{d x_{1}(t)}{d t}=-k_{1} x_{1}(t) \\
& \frac{d x_{i}(t)}{d t}=k_{i-1} x_{i-1}(t)-k_{i} x_{i}(t), \quad 2 \leq i \leq n .
\end{aligned}
$$

Where, $x$ is concentration of pollutant in the cascade; $n$ is number of reservoirs in the cascade; and $k=\frac{f}{V_{i}}$. Equation (3.2) indicates that fresh water enters the initial water reservoir so that the particle concentration $x_{1}(t)$ due to a single source is negative. Equation (3.3) is a global ordinary differential equation which describes the change in concentration between two consecutive reservoirs, $(i-1)^{\text {th }}$ and $i^{\text {th }}$ in the cascade through $n$ reservoirs. It should be noted that in its application, such as on the dynamics of river flow involving pollution, the system of ordinary differential equation (equation 3.3) provides net concentration $x(t)$ of particles only between two progressive adjacent cascade reservoirs, $i^{t h}$ and $(i+1)^{t h}$.

\subsection{Equation for Single Species Population model with delay}

In modeling population dynamics, Erwin (2005) provides DDE model for a single species population as:

$$
\frac{d x}{d t}=b x(t-h) e^{-a x(t-h)}-d x(t)
$$

If $b>d$, then the equation (3.4) will have nontrivial steady states. The study considered the case of equation (3.4) when the average death rate $d$ is constant, and the average birth rate $b$ has an exponential decaying factor. Examples where this type of model has been applied include 'Nicholson's blowfly data' on laboratory populations of blowflies, in which the essential features of the model are two functions relating fecundity and egg-to-adult survival to the amount of food available to each individual. The model describes thresholds representing maintenance requirements in the two functions, $b$ and $d$, together with a time delay $\tau$ due to the development that cause cycling in the blowfly populations. Similar thresholds and time delays occur in more complex biological systems with cycling in closely interacting populations of predators and prey (Gourley \& Kuang, 2004).

\section{Results and Discussions}

\subsection{Formulated Discrete Time Delay Mathematical Model}

Steady, uniform and three-directional flow in a river, stream or generally sub-surface water systems with cascade geological setting was considered. First, equations (3.2) and (3.3) derived from mixing-problem processes in a controlled set-up of water tanks (Antonin, 2013) were used to provide a conceptual framework (methodology) for natural sub- 
surface water reservoirs of cascading geological setting. Second, since pollution concentration in the reservoirs is never instantaneous, the equations were modified to include lag or delay time $\tau$. This modification considered concentration $x(t)$ of a pollutant as a reducing or decaying factor so that the final model equation for particle transport would conform to equation (3.4) from population dynamics. To achieve this, the changes in concentration of particles in a particular reservoir were analogized to the dynamics of population change of a single species in an ecological niche, and therefore the following derivation was resourceful:

Considering a simple population growth model with unlimited resources, the changes in population size $N(t)$ at any given time can be represented in global variables as,

$$
\frac{d N}{d t}=(B-D) N(t), \quad B, D>0
$$

The constants $B$ and $D$ are the birth and death rates, respectively. This model assumes that birth and death rates are directly commensurate! Natural realities in biological populations indicate that these rates are never commensurate. Can this model, therefore, be improved to simulate such realities in a population? Let $N(t)$ be the number of adults. The babies of the population species should take time $\tau$ to grow up and with probability $\rho$ to make it to full adults. Then,

$$
\frac{d N}{d t}=B \rho N(t-\tau)-D N(t)
$$

This is a delay differential equation (DDE), named Hayes Equation (Erwin, 2005). In particular, by considering the independent variables $B, D, \rho$ and $\tau$, equation (4.2) is similar to equation (3.4).

\subsubsection{Model Formulation}

The model DDE equation was provided in the form of equation (3.4) from population dynamics through equation (4.2) and then parameter setting mathematical approach was used to investigate the stable states of the resulting model equation. This approach involved combinations of a range of values of the time lags $\tau$ and coefficients $\beta, \partial$ in the DDE equation so as to obtain a steady stable (or unstable) state.

Following the derivation of Hayes equation in equation (4.2), equation (3.3) was modified to the following model equation.

$$
\frac{d x(t)}{d t}=\beta_{i}(t)[x(t-\tau)] e^{-A x\left(t-\tau_{i}\right)}-\partial_{i}(t) x(t), \quad i=1,2,3, \ldots, n
$$

Where $i$ is the position of reservoir in the cascades, $x(t)$ is the concentration of pollutant species in sub-surface water reservoir, $\tau_{i}$ is the time lag of pollutant flow in the cascade reservoir and is considered to be of unique value for a particular reservoir so that $\tau_{1}<\tau_{2}<,,,<\tau_{n}$, for reservoirs $1,2,3, \ldots, n$. This specifies equation (4.3) as DDE with multiple discrete delays. The parameters $\beta, \partial$ are time-dependent coefficients, so that equation (4.3) is non-autonomous.

The coefficient $\beta(t)$ is termed as the incursion term and represents pollutant particles that enter the $i^{t h}$ reservoir with a rate of flow $f_{i-1}$, which is the rate of flow in the previous adjacent reservoir. $\beta(t)$ is considered a continuous, positive, decreasing function, so that the average concentration rate $\left(\frac{d x}{d t}\right)$ of the pollutant decreases with increased number of cascade water reservoirs. Similar consideration on this coefficient has been done for density-limited growth and the logistic models in population dynamics (Erwin, 2005).

The coefficient $\partial(t)$ is called the dilution term which accounts for the spreading rate (flux divergence rate) of pollutant particles and therefore reducing the average concentration per unit volume of particles flowing in the $i^{\text {th }}$ reservoir. This term is comparable to the death rate $d$ of a single species in population dynamics, which is increased by factors like intraspecific competitions for survival. For example, in Nicholson's blowfly model (Erwin, 2005), $d$ is considered constant and represents constant per capita death rate. Similarly, in applications of equation $(4.3), \partial_{i}$ in the $i^{\text {th }}$ reservoir is considered constant in that particular reservoir. 
The invading particles in the $i^{\text {th }}$ reservoir do not mix up instantaneously with water, but rather takes time $\tau_{i}$ in the mixing process. Some of these particles will be harbored in the reservoir due to chemical, biological and physical processes and therefore may or may not eventually flow into the $(i+1)^{\text {th }}$ reservoir. With reference to equation (4.2), Hayes' equation, this possibility is represented in the model equation (4.3) by the mathematical probability $\rho(t)=e^{-A x\left(t-\tau_{i}\right)}$. This term is attached to the incursion term $\beta(t)$ to mathematically account for the progressive exponentially reducing (decaying) incursion rate of pollutant particles in the cascades. The constant $A$ is mathematically used as a scaling factor and is assigned a negative value so that the exponential term can reciprocally provide the decaying factor in the incursion term.

The following mathematical definition was provided for particle concentration $x(t)$, so as to specify equation (4.3) for a solution which is bounded for each water reservoir in the cascade.

\section{Definition}

If $\frac{d x(t)}{d t}=F\left(t, x_{t}\right), x(t) \in \mathbb{R}^{n}$, where $x_{t}(t+\theta), \theta \in(-\infty, 0]$ represent a trajectory of the solution in the past, then $F$ represent a functional operator from $\mathbb{R} \times C^{1}$ to $\mathbb{R}^{2}$ (Humphries T., 2016).

The consequence of this definition is to specify the model equation (4.3) as a functional delay differential equation with the following properties:

- The delays $\tau_{i}$ are discrete with $\tau_{1}<\tau_{2}<\cdots<$, $\tau_{n}$ for every $\tau \in \mathbb{R}$.

- Concentration, $x(t) \in \mathbb{R}^{n}$.

- $X_{(t)}=\{x(\tau): \tau \leq t\}$

- Initial conditions: $\emptyset:[-\tau, 0] \rightarrow \mathbb{R}^{n}$.

- The characteristic equation is transcendental.

\subsubsection{Linearized Stability of Model Equation (4.3)}

This sub-section was aimed at setting conditions for the determination of values of the coefficients $\beta$ and $\partial$ as a pair in equation (4.3) that will always give rise to a stable (or unstable) state on application of the DDE. This was achieved through a criterion derived to evaluate a critical delay, $\tau_{c}$ from a suitable combination of values of $\beta$ and $\partial$. The delays, $\tau_{i}$ will then be determined based on the value of $\tau_{c}$ so as to sustain stability of resulting DDE.

The study derived a lot of insight from stability analysis of equilibria. An equilibrium point in the state space was considered for which $x(t)=\bar{x}$ is a solution of equation (4.3) for all $t$, so that equilibrium points satisfy.

$$
f(\bar{x}, \bar{x}, \bar{x}, \ldots, \bar{x})=0
$$

In principle, when a solution or solutions of the characteristic equation has positive real part, then the equilibrium point is unstable. If they all have negative real parts, the equilibrium point is stable. When the leading characteristic values are zero, then the stability is unreduceable to linear order.

It should be realized that the basic properties of this model are the type of functions $\beta$ and $\partial$ which might lead to the existence of periodic solutions of equation (4.3). The study specified the use of the case $\beta(t)=\beta_{i} e^{-A x\left(t-\alpha_{i}\right)}$ and $\partial(t)$ constant in each of the cascade reservoir in cascade, so as to prove the existence of the periodic solution of the model equation.

\subsubsection{Preliminary Analysis of Model Equation}


An analytical approach was provided to establish basic properties of solutions to the equation (4.3) under the following projections:

- Given positive initial data of concentration $x(t)$, solutions remain positive in all time $t$. This positivity of solutions is important in modeling physical and biological phenomena because negative solutions (values) will be meaningless.

- Solutions are bounded and eventually uniform bounded regardless of initial data. Pollution in a flow is characterized with particles moving along a concentration gradient. The rate of the mixing process of pollutant particles is affected by initial concentration. Such rates are expressed in terms of time delays in any dynamics of flow. Eventually a uniform particle flow is attained for every initial concentration when particles concentration is minimum constant or otherwise zero in the long run, or maximum levels.

- Determine steady state solutions and their stability:

-identify delays that cause instability to non-trivial steady states of equation (4.3). If the non-trivial steady states exist for a solution, then there is a critical delay corresponding to every such non-trivial steady state. -propagate the existence of periodic solutions. DDEs that model physical dynamics exhibit oscillatory phenomena whose analytical results provide periodic solutions (Erwin, 2005).

To achieve the above set projections the following analytical approach provided in Erwin (2005) was applied.

The study relied on the condition that $\beta>\partial$, so as to be lead by the case of Theorem (4.1) below, where the nontrivial steady state exists. Besides, for particles to flow in a water reservoir, their rate of 'production' must be higher than their 'death' rates.

\section{Theorem (4.1)}

Let $\beta$ and $\partial$ be positive functions. Suppose that their exists an $\bar{x} \in x$ such that:

$\operatorname{sign}(\beta(x)-\partial(x))=-\operatorname{sign}(x-\bar{x})$, and $\beta^{\prime}(\bar{x})<\partial^{\prime}(\bar{x})$. Then $\bar{x}$ is a positive steady state, and the trivial steady state is unstable. If $\beta^{\prime}(\bar{x}) \bar{x}>-2 \partial(\bar{x})-\partial^{\prime}(\bar{x}) \bar{x}$, then $\bar{x}$ is linearly stable for all $\tau$. Otherwise, there exists a $\tau_{i}>$ 0 such that $\bar{x}$ is stable for $\tau_{i}<\tau_{c}$, and unstable for $\tau_{i}>\tau_{c}$ (Erwin, 2005).

Using Theorem (4.1) above, the particulars of the case $\beta>\partial$ were analyzed, which is a necessary condition for particle flow to occur and for completeness of the mixing process within a water reservoir. The analysis leads to a condition for the boundedness of the delays $\tau_{i}$ in SWR.

From Theorem (4.1) and considering the case $\beta>\partial$, the non-trivial steady state occurs whenever,

$$
\beta=\partial e^{A \bar{x}}
$$

or,

$$
\bar{x}=\frac{1}{\mathrm{~A}} \ln \frac{\beta}{\partial}
$$

Then, according to Theorem (4.1), $\bar{x}$ is stable for all $\partial$ if and only if

$$
\frac{d}{d x}\left[\beta e^{-A x}\right]_{x=\bar{x}}>-2 \frac{\partial}{x}
$$

This is equivalent to the condition that $\beta<\partial e^{2}$, which is a contradiction to the necessary requirement that $\beta>\partial$ for particles to flow in solution.

This condition was used to validate model equation (4.3) by determining a pair of measured values $(\beta, \partial)$ from the dynamics of water flow involving change of concentration of pollutant particles, so that $\beta>\partial$ for unstable solution that is essential for the periodic property of the DDEs. So as to determine the range of values of delay $\tau$ that would 
give rise to a stable or unstable state solution of equation (4.3) in its application, the following criterion based on Theorem (4.1) was applied (Erwin, 2005).

The condition $\beta>\partial e^{2}$ was considered for a steady state of equation (4.3). Laplace transforms show that all analytic solutions of autonomous constant delay DDEs are exponentials (Humphries T., 2016). To obtain a characteristic equation, therefore, the simple exponential $e^{k t}$ may be chosen and posted in equation (4.3) to get,

$$
k e^{k t}=\beta e^{k(t-\tau)}\left[e^{-A e^{k(t-\tau)}}\right]-\partial e^{k t}
$$

Dividing by $e^{k t}$ and rearranging gives a transcendental exponential.

$$
k+\partial\left(\ln \frac{\beta}{\partial}-1\right) e^{-k \tau}+\partial=0
$$

Taking $\sigma=\left(\ln \frac{\beta}{\partial}-1\right)$ leads to the roots:

$$
x(k)=-\partial \sigma e^{-k \tau}-\partial=0
$$

The roots $k$ have two cases to address on the application of equation (4.3). When there is no delay, like in the case of flow without mixing processes, $\tau=0$ resulting to simple polynomial, $k=-\partial \sigma-\partial$, which is insignificant as far as pollution is concerned. But, if $\tau>0$, then $k=k_{1}+k_{2} i$, which is a complex root. Taking the real and imaginary parts:

$$
k_{1}=-\partial \sigma \cos \left(k_{2} \tau\right)
$$

and,

$$
k_{2}=-\partial \sigma \sin \left(k_{2} \tau\right)
$$

Squaring and summing up yields

or,

$$
k_{1}^{2}+k_{2}^{2}=\partial^{2} \sigma^{2}
$$

$$
k_{1}=k_{2}\left(\sigma^{2}-1\right)^{1 / 2}
$$

Rewriting the real and imaginary parts of the roots of the characteristic equations,

$$
\cos \left(k_{2} \tau\right)=-\frac{1}{\sigma}<0
$$

and

$$
\sin \left(k_{2} \tau\right)=\frac{\left(\sigma^{2}-1\right)^{1 / 2}}{\sigma}>0
$$

Thus, there exists a critical delay $\tau_{c}$, at which an eigen value crosses into the right half-plane, $k_{2} \tau_{c} \in\left(\frac{\pi}{2}, \pi\right)$, and the critical delay is given by

$$
\tau_{c}=\frac{1}{\partial\left(\sigma^{2}-1\right)^{\frac{1}{2}}} \cos ^{-1}\left(-\frac{1}{\sigma}\right) .
$$




\subsection{Time $t$ Necessary for Transport of Pollutants through Cascaded SWR}

The formulated model (equation 4.3) was solved in matlab dde23 software to obtain time $t$ necessary for transport of single species pollutant in cascading sub-surface water reservoirs (SWR). It should be realized that the basic properties (such as linearity, stability and oscillatory) of equation (4.3) are determined by the types of functions $\beta$ and $\partial$.

The time series solutions for the system of equation (4.3) were generated graphically through Matlab by assigning paired values of the coefficients $\beta_{i}$ and $\partial_{i}$ in a particular reservoir, that were predetermined for the boundedness of the corresponding delay $\tau_{i}$ using equation (4.17). These time series curves displayed similar tendencies downstream from the point source to the last reservoir in the cascade, and therefore they constitute characteristic curves. Curves resulting from the dynamics in reservoirs nearer the point source have longer times to cover than those far from the point source. The intensity (magnitude) of the point source pollutant also affect the duration of the curves. Higher levels of the pollutant concentration at point source cause longer amplitudes, and vice versa. One remarkable result of the DDE model was obtained on comparing its results to the results of ODEs it modifies under the same dynamic system of flow. The time series solutions obtained for the DDE model displayed longer curves than the ODE counterparts in the same reservoir for the same single species pollutant.

Therefore, the time $t$ necessary for transport of pollutant in cascading SWR can be evaluated numerically using the DDE model. Further, since the process of water reservoir pollution is not instantaneous, but rather takes time to occur, the model provides the time inherent for this process as time delays, $(t-\tau)$.

\subsubsection{Assumptions for the Model}

The following assumptions were found necessary for the formulation and application of the model:

I. Flow in sub-surface water reservoirs was assumed steady, uniform and uni-directional.

II. Water free from pollutant-species flows into the first reservoir in the cascade and pollutant in solution flows out of every reservoir at the same rate, ensuring that the amount of fluid flow remains constant in the cascade. This way, the time $t$ was determined that accounted for movement of pollutant through the cascades since its injection into water reservoirs through a point source until maximum concentration is reached in the whole system of water flow.

III. Only a single point source of pollutant species into water system was considered.

IV. It was assumed that mixing of pollutant particles in water (in every reservoir) was perfect and complete and that the flow rates (in and out) of reservoir were well observed (measurable).

\subsection{Model Validation}

The described mathematical model was validated on the dynamics of flow involving pollution on river Nyakomisaro in Kisii town. The river is of shallow water with average depth 0.5 metres (at minimum during dry season) and $1-2$ metres (at maximum during high rainfall) and average width below 3 metres. The river transverses densily populated Kisii town and therefore is characterized with several point sources of pollution from the municipal solid and dissolved wastes flowing into the river through its banks. Concentration, for pollutant species were selected at particular point sources along the river banks.

From the studies conducted on the river involving pollution (Omoko, Onyatta, Nyabaro \&Kenanda, 2016), the initial pollutant concentrations at the identified point sources for SECTIONS A, B, and C were taken as 2.228ppm, $4.50 \mathrm{ppm}$ and $6.80 \mathrm{pmm}$, respectively.

\subsubsection{Determination of Flow Volume}

Each reservoir volume $V_{i}$ was calculated from the length $(L)$ and average cross-sectional area $(A)$ for every $i^{t h}$ reservoir as:

$$
V_{i}=\left(A_{i} L_{i}\right)
$$


Where, $A=($ depth $\times$ width $)$. Measurements of depth, width and length were take downstream at every discretized endpoint of a reservoir using meter-rule and string.

\subsubsection{Discretization of Time-Lags}

In the physical applications of the DDE equation (4.3), the lags are parameters distinctively calculated as a ratio of the cascade length to total length of the cascade zone:

$$
\tau_{i}=\frac{L_{n}}{L_{T}}
$$

where, $L_{n}=\sum_{1}^{n}\left(L_{i}\right)$, for $i=1,2,$, , $n$. These proportions were necessary to maintain that $\tau_{1}<\tau_{2}<$, , , $<\tau_{n}$, so that the time lags are distinctively discrete and in series.

Table 4.1 Boundedness of Delays, $\tau_{i}$

\begin{tabular}{|ccc|}
$\boldsymbol{i}^{\text {th }}$ Reservoir & $\begin{array}{c}\text { Length of Reservoir, L(m) } \\
\text { For Sections: } \\
\text { 'A, B and C' }\end{array}$ & $\boldsymbol{\tau}_{\boldsymbol{i}}>\boldsymbol{\tau}_{\boldsymbol{c}}$ \\
\hline $\mathbf{0}$ & 20 & 0.000 \\
\hline $\mathbf{1}$ & 50 & $0.091>0.064$ \\
\hline $\mathbf{2}$ & 60 & $0.200>0.166$ \\
\hline $\mathbf{3}$ & 80 & $0.345>0.286$ \\
\hline $\mathbf{4}$ & 100 & $0.527>0.497$ \\
\hline $\mathbf{5}$ & 120 & $0.745>0.623$ \\
\hline
\end{tabular}

Table 4.2 Kinetic Parameters from River Nyakomisaro

\begin{tabular}{|c|c|c|c|c|}
\hline$i^{\text {th }}$ Reservoir & \multicolumn{2}{|c|}{$\begin{array}{l}\text { Flow rate } f_{i-1}(\mathrm{~m} / \mathrm{s}) \\
\text { At Sections: }\end{array}$} & \multicolumn{2}{|c|}{$\begin{array}{l}\text { Reservoir Volume, } \\
V_{i}\left(m^{3}\right)\end{array}$} \\
\hline 0 & 1.55 & 3.20 & 400,000 & 220,000 \\
\hline 1 & 1.50 & 2.96 & 536,000 & 145,000 \\
\hline 2 & 1.10 & 2.66 & 250,000 & 156,800 \\
\hline 3 & 0.92 & 2.35 & 400,000 & 172,000 \\
\hline 4 & 0.80 & 1.90 & 381,700 & 182,400 \\
\hline 5 & 0.60 & 1.76 & 402,000 & 188,000 \\
\hline 6 & 0.50 & 1.62 & 322,000 & 192,000 \\
\hline
\end{tabular}

\subsubsection{Coefficients for Model Equation}

Using the results in table 4.2, the incursion term $\beta_{i}(t)$ to every cascade was calculated from the relation:

where,

$$
\beta_{i}(t)=\frac{f_{i-1}}{V_{n}}
$$

$$
V_{n}=\sum_{1}^{n}\left(V_{i}\right), \quad i=1,2,,, n, .
$$

The computed results for the six cascade zones were recorded in table $\mathbf{4 . 3}$ below. 
Table 4.3 Values of $\beta, \partial$ and Initial Concentration, $X(t)$

\begin{tabular}{|ccccc|}
\hline $\begin{array}{l}\boldsymbol{i}^{\boldsymbol{t h}} \\
\text { Reservoir }\end{array}$ & $\mathrm{A}$ & $\begin{array}{c}\boldsymbol{\beta}_{\boldsymbol{i}} \\
\left(\times \mathbf{1 0}^{-\mathbf{6}}\right) \\
\left(\boldsymbol{m}^{\mathbf{2}} \boldsymbol{s}\right)^{-\mathbf{1}}\end{array}$ & $\boldsymbol{\partial}_{\boldsymbol{i}}$ \\
\hline $\boldsymbol{X}(\boldsymbol{t})$ & $\mathbf{2 . 2 2 8}$ & $\mathbf{4 . 5 0 0}$ & $\mathbf{6 . 8 0 0}$ & \\
\hline $\boldsymbol{i}$ & 2.800 & 2.800 & 0.204 & $\mathbf{0}$ \\
\hline $\boldsymbol{i}+\mathbf{1}$ & 1.399 & 1.399 & 0.169 & $1 / 4$ \\
\hline $\boldsymbol{i}+\mathbf{2}$ & 0.776 & 0.776 & 0.136 & $1 / 64$ \\
\hline $\boldsymbol{i}+\mathbf{3}$ & 0.509 & 0.509 & 0.104 & $1 / 256$ \\
\hline $\boldsymbol{i}+\mathbf{4}$ & 0.304 & 0.304 & 0.093 & $1 / 1024$ \\
\hline $\boldsymbol{i}+\mathbf{5}$ & 0.218 & 0.218 & 0.084 & $1 / 4096$ \\
\hline
\end{tabular}

The coefficient $\partial_{i}(t)$, for every cascade reservoir was determined using the method of 'serial dilution'. The dilution factor (DF) for each step was computed as the ratio of the total volume, $V_{T}$ (obtained by summing up $V_{i}$ for $i=$ $1,2,3, \ldots, n$ where $n$ is the number of zones in the cascade) to $V_{0}$, volume of water (assumed to be free of single species pollutant) entering the cascade zone. Thus,

$$
D F=\frac{V_{t}}{V_{0}}=\frac{1630800}{400000} \approx 4
$$

$V_{0}$ is the volume of pre-cascade reservoir which is determined just before entering the first cascade reservoirs. Using this approach, and with the assumption that 'freshwater' enters the contaminated cascade through the first reservoir (at the point source), the dilution term $\partial_{i}$ was computed as the DF of the system. Thus the DF up to sixth reservoir was reached as:

$$
\frac{1}{4}, \frac{1}{16}, \frac{1}{64}, \frac{1}{256}, \frac{1}{1024}, \frac{1}{4096}
$$

This computation was necessary to show that the concentration of the pollutant reduces downstream leading up to the sixth reservoir where it is 4096 times less than the original 'undiluted' solution inside the first reservoir.

\subsubsection{Particle Flow for $\tau=0$}

The model equation (4.3) was transformed into its ordinary differential equation counterpart by setting the delays $\tau_{i}=0$, which resulted to:

$$
\frac{d x}{d t}=\beta_{0} x(t) e^{-A x(t)}-\partial_{i} x(t)
$$

The coefficient $\beta_{0}$ represents the rate per unit volume (flow flux) at which pollutant particles are entering the $i^{\text {th }}$ reservoir at the boundary point. Because no history of particle flow is required to solve ODEs, a solution for equation (4.3) is guaranteed at the entry boundary point of each reservoir. The consequence of the choice of this boundary point as an ordinary point for the ODEs is to calculate $\beta_{0}$ using the relation:

$$
\beta_{0}=\frac{f_{i}}{V_{i}}
$$

Where, $f_{i}$ and $V_{i}$ represents rate of flow (velocity) of pollutant particles and the water volume in the $i^{\text {th }}$ reservoir, respectively. It turns out that $\beta_{0}$ for a given reservoir is a constant defined at the commencement of the flow process of pollutant particles in solution for that particular reservoir. This specification of $\beta_{0}$ executes the mixing process of pollutant particles in water flow as 'instantaneous', so that particle concentration in all other points in a reservoir is 
uniform without any time elapse. The coefficient $\partial_{i}$ remains the serial dilution factor in each reservoir and was computed using relation (4.22). Graphical displays of time series solutions for each reservoir in the cascade were demonstrated in comparison for both model equation (4.3) and ODE equation (4.24) in the following section.

\subsubsection{Validation of Results and Discussion}

When the model equation (4.3) was ran using MATLAB for the data sets for $\beta_{i}, \partial_{i}, \tau_{i}$ where $i=1,2,3, \ldots, 6$ obtained from river Nyakomisaro, it produced graphical view that varied from the paired parameter values for $\beta, \partial$ and $\tau$ in each reservoir, downstream. From the graphs 4.1(a), 4.1(b) and 4.1(c), it was realized that as the values of the parameters $\beta, \partial$ become smaller, the curve is less clear and has shorter times to cover than when the parameter values are larger.

This also agreed with the pattern of the natural phenomenon in mixing-problems that the rate of particle flow decreases with decreased concentration, a condition described as 'concentration attenuation'. Therefore, particle migration is faster and more feasible at early stages (front reservoirs) of pollutant incursion into water system than when particles must have stayed (rare reservoirs) in the system.

Further, it was observed (from the graphs in Figures 4.1(a) to 4.1(c)) that as the values of the parameters $\beta, \partial$ were varied downstream in a river flow, the resulting curves change pattern. The dips (troughs) of the curves change gradually and eventually to linear with zero slopes.

\section{Figures 4.1 Time Series Trajectories for Particle Flow in Reservoirs}

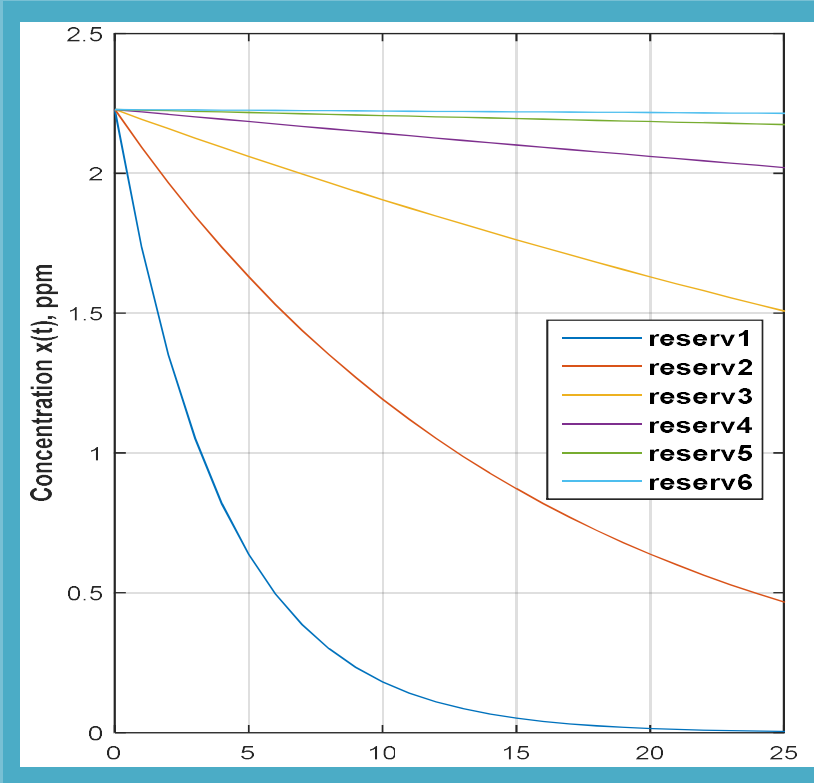

$t$ (min)

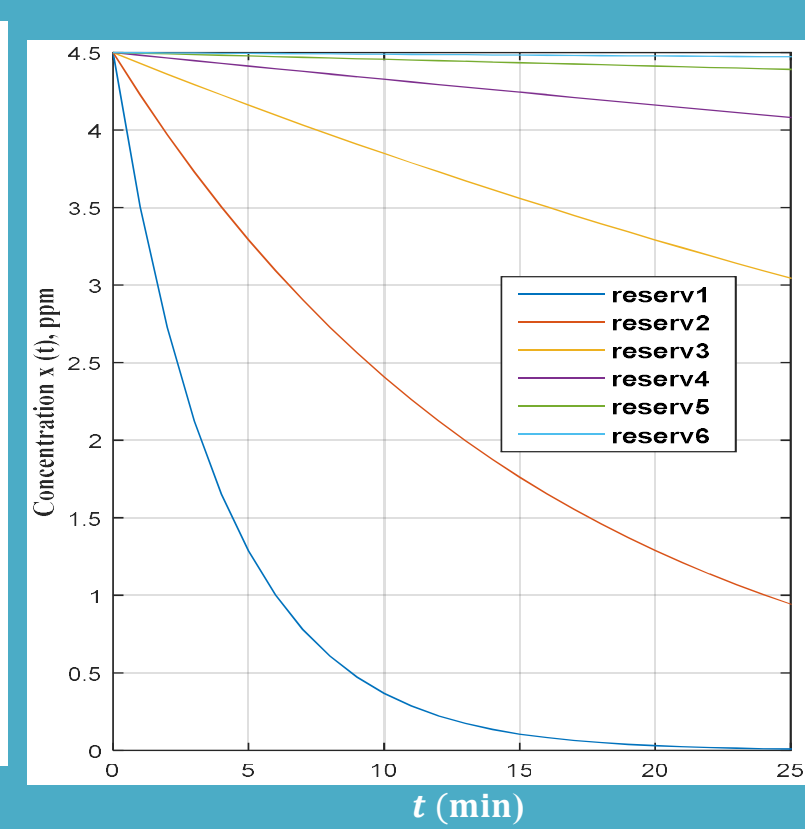

Figure 4.1(b) plot of $x(t)$ vs $t$ for 'SECTION B' 


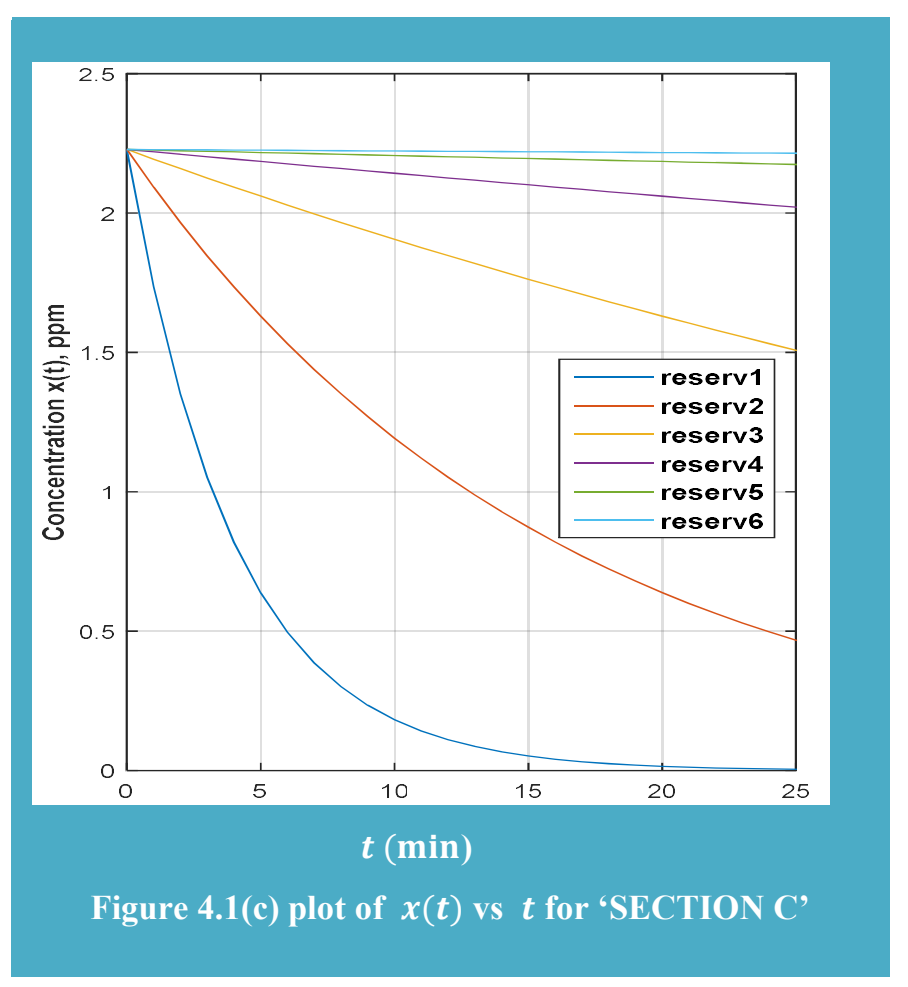

Figures 4.3 Graphs of $\boldsymbol{x}(\boldsymbol{t})$ vs $\boldsymbol{t}$ for Comparison of DDEs and ODEs in Reservoirs

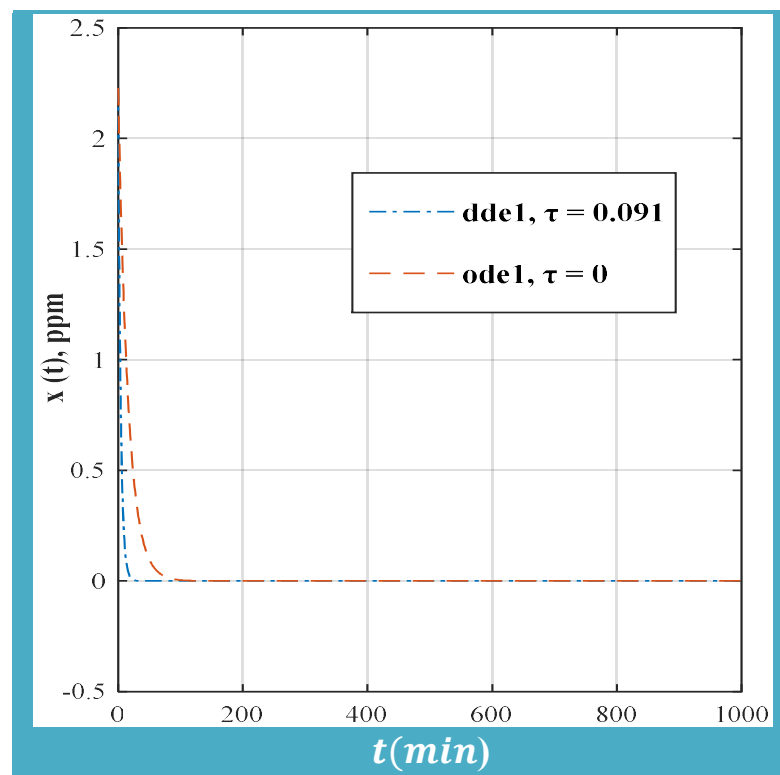

Figure 4.2(i) Reservoir 1 of 'SECTION A'

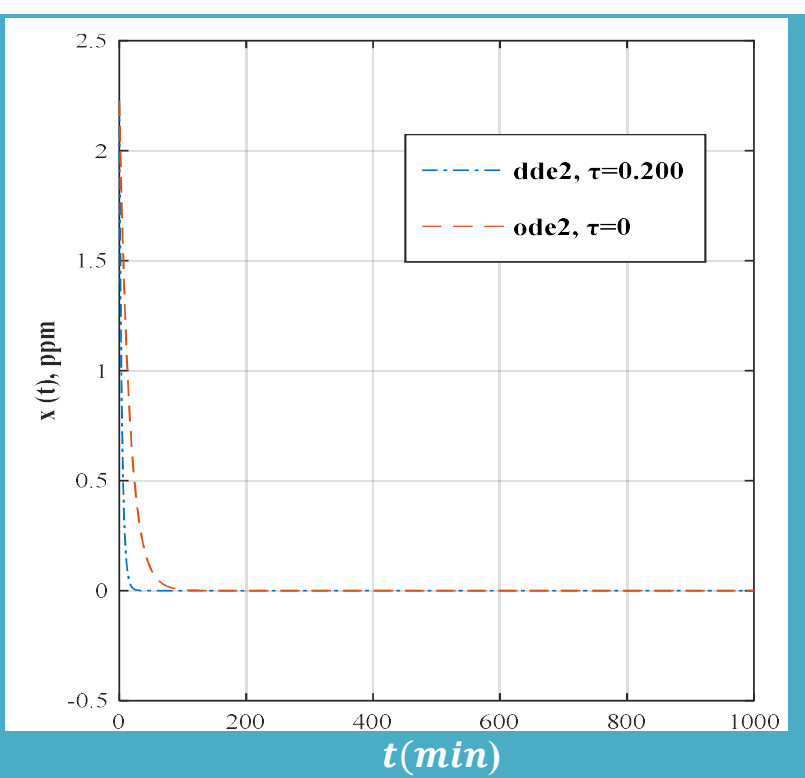

Figure 4.2(ii) Reservoir 2 of 'SECTION A' 


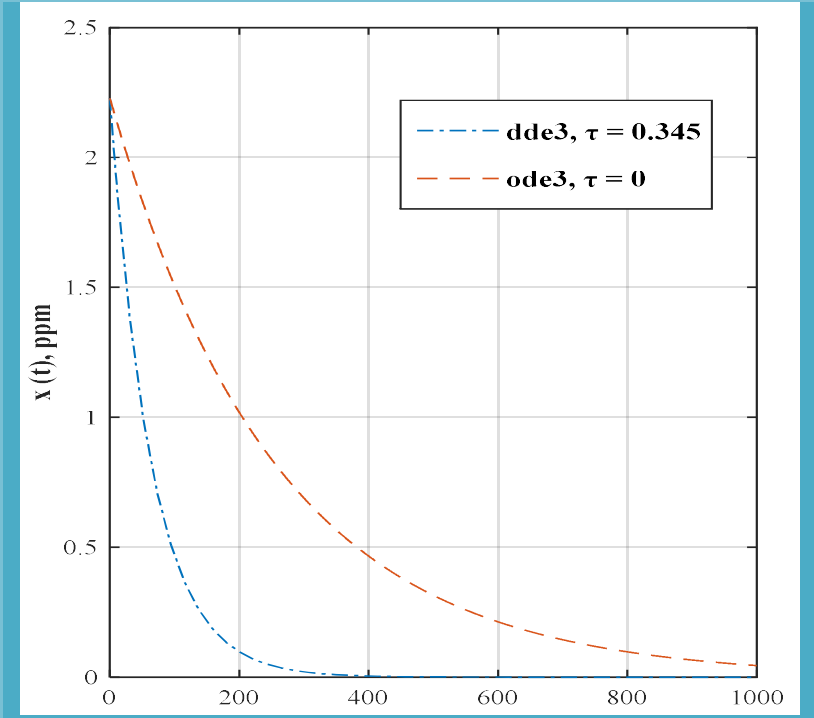

$t(\min )$

Figure 4.2(iii) Reservoir 3 of 'SECTION A'

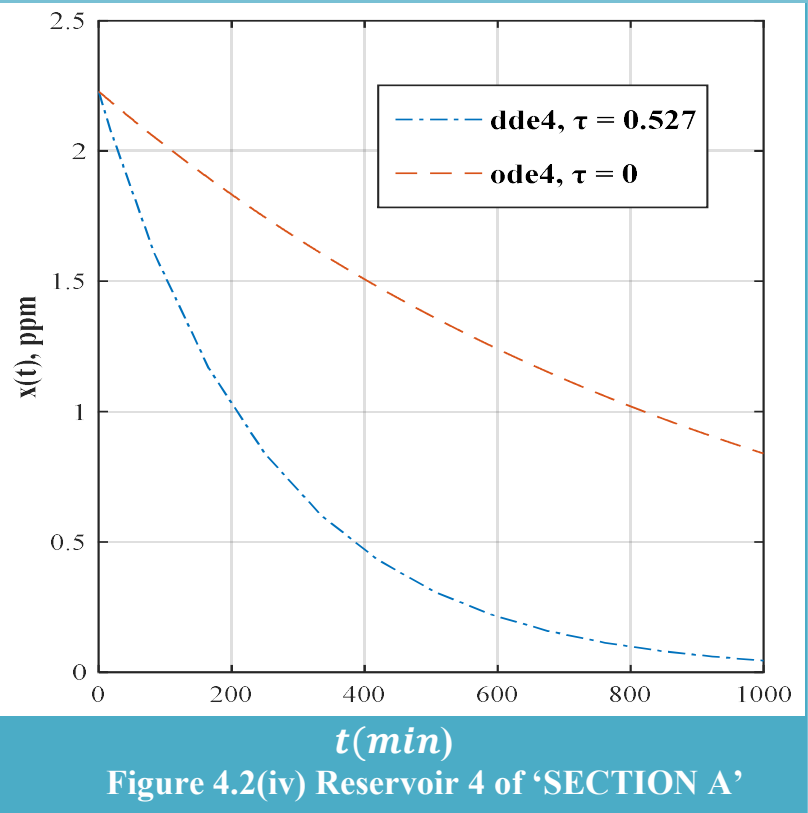

Figure 4.2(iv) Reservoir 4 of 'SECTION A'

From the graphs given in figures 4.2(i) to 4.2(iv), it was observed that for the same reservoir, under similar physical properties, an ODE curve has shorter times to cover than its DDE curve counterpart. It implies that if the time delays in the mixing processes are not accounted for in particle (pollutant) transport in water reservoirs, and since mixing processes are never instantaneous, then the time required for transport of a species of pollutant in the water reservoir is underestimated. The time series solution that include delays generated by model equation (4.3) offers a better approximate value of the time necessary for particle flow in cascade sub-surface water reservoirs than mathematical models without delays (Antonin, 2013).

\section{Conclusion}

The numerical model formulated in this study describes the kind of time delays observed in mixing-problems that govern the dynamics of water pollution in sub-surface water reservoirs. The results from the validation of the model, displayed in figures (4.1) and (4.2), imply that if the average time elapsing for mixing-process of pollutant particles within a water reservoir are accounted for (in models with time delays), and if the duration of the particle movement within the reservoir up to their possible maximum accumulation in water systems are approximately negative exponential in distribution, then such a model is more appropriate (than models without delays) to estimate time evolution of particle transport in water reservoirs.

The model was applied on three stretch sections on river Nyakomisaro in Kisii town, labeled 'SECTION A, SECTION B and SECTION C'. These three sections were identified by recognizing possible potential pollutants entering the river at particular points (point sources). The three stretches were of equal lengths and each was partitioned into six cascade zones. Physical parameters of the river at these sections were measured and the coefficients of model equation (4.3) were evaluated. It turned out that the graphical views generated displayed similar tendencies (characteristics) even though initial values of concentration $x(t)$ were different for every potentially recognized single species pollutant chosen for each section. The graphical comparisons for three sections with different starting points (but with constant delays) were given in figures (4.1). 


\section{Recommendations}

More validation of model equation to different natural river flow systems is recommended.

\section{References}

Anderson, M.P., Woessner, W.W., Hunt, R.J. (2016). Applied Groundwater Flow Modeling: Simulation of Flow and Advective Transport. Washington, D.C., 37-45. National Academy Press, Washington D.C., 37-45.

Antonin, S. (2013). Mixing-Problems with Many Tanks. The Mathematical Association of America. Charles University, Sokolovska 83, Czech Republic.

Bartran, J., Rees, G. (2000). Mornitoring Bathing Waters: Practical Guide to the Design and Implementation of Assessments and Monitoring Programmes. London, E \& FN Spon. Published on behalf of WHO, Commission of Communities and EPA.

Erwin, J.F. (2005). Delay Differential Equation Models in Mathematical Biology. The University of Michigan.

EPA., (2007). Groundwater Flow and Fate and Transport Modeling. Technical Guidance Manual Hydrogeological Investigations and Groundwater Monitoring (TGM). Chapter 14. Columbus.

Gourley, S.A., Kuang, Y., (2004). A Stage Structured Predator-Prey Model and its Dependence on Maturation Delay and Death Rates. Journal for Mathematical Biology, 49:188-200.

Humphries, A.R., DeMasi, O.A., Magpantay, F.M.G., Upham, F. (2012). Dynamics of a DDE with Multiple StateDependent Delays: Discrete and Continuous Dynamical Systems. 32(8);2701-2727

Humphries, T., (2016). Continuation Methods in Dynamical Systems. Reglan, New Zealand.

Nick, A., Leif, W.,Foppen. J., Kulabako, R., Xavier, D.L.,Samwel, M., Wagner, F., (2012). Sustainable Sanitation and Groundwater Protection. Fact sheet of Working Group 11, Sustainable Sanitation Alliance (SuSanA).

Okwoyo, J.M., (2011). Synchronization in a Network of Oscillators with Delayed Coupling. JKUAT, Department of mathematics, Kenya. Available at ir.jkuat.ac.ke>bitstreams>handle, Thesis, 15(2) 2011,113-128.

Omoko, Onyatta, Nyabaro and Kenanda (2016). Levels of Metal Pollutants in Water from Nyakomisaro Stream through Kisii Town. International Journal of Science and Research (IJSR). Available at https://www.ijsr.net>DOI:10.21275/i7.ART 2016141.

Patil, S.B., \& Chore, H.S., (2014). Contaminant Transport through Porous Media. An Overview of Experiments and Numerical Studies Advances in Environmental Research. Volume 3, No. 1, (2014), page 45-69. Department of Civil Engineering, DaltaMeghe College of Engineering, Airoli, Navi Mumbai, India.

Roussel, M. R., (2005). Delay-Differential Equations. Retrieved from People.uleth.ca/ roussel/nld/delay. Pdf).

Shampine, L.F.,(2001). Solving Delay Differential Equations in MATLAB. Applied Numerical Mathematics, 37, 441-458. 